\title{
Effect of Different Types of Dietary Fatty Acids on Subclinical Inflammation in Humans
}

\author{
I. KRÁLOVÁ LESNÁ ${ }^{1}$, P. SUCHÁNEK ${ }^{1}$, E. BRABCOVÁ ${ }^{2}$, J. KOVÁŘ ${ }^{1}$, H. MALÍNSKÁ ${ }^{3}$, \\ R. POLEDNE ${ }^{1}$
}

${ }^{1}$ Laboratory for Atherosclerosis Research, Institute for Clinical and Experimental Medicine, Prague, Czech Republic, ${ }^{2}$ Immunology Department, Institute for Clinical and Experimental Medicine, Prague, Czech Republic, ${ }^{3}$ Department of Metabolism and Diabetes, Institute for Clinical and Experimental Medicine, Prague, Czech Republic

Received July 31, 2012

Accepted September 6, 2012

On-line December 13, 2012

\begin{abstract}
Summary
Replacing SAFAs (saturated fatty acids) for vegetable PUFAs (polyunsaturated fatty acids) has a well documented positive effect on the lipoprotein pattern while the direct effect of dietary fatty acids composition on systemic inflammation remains to be proven. In well controlled randomised cross-over study with 15 overweight/obese postmenopausal women, the effect of dietary switch on systemic inflammation was investigated. A two 3 weeks dietary period either with predominant animal fat (SAFA, 29 caloric \% SAFA) or vegetable fat (PUFA $25 \%$ caloric \% PUFA) were interrupted by wash-out period. The expected increasing effect on SAFA diet to LDL-C (low density cholesterol) and opposite effect of PUFA diet was documented following changes in fatty acid spectrum in VLDL (very low density cholesterol) particles. The switch from SAFA diet to PUFA diet produced a significant change of CRP (C-reactive protein) concentration $(p<0.01)$ whereas similar trend of IL-18 did not reach statistical significance. In this study, previous in vitro results of different SAFA and PUFA proinflammatory effects with well documented molecular mechanisms were first proven in a clinical study. It could be stated that the substantial change of dietary fatty acid composition might influence proinflammatory effect in addition to traditional cardiovascular risk factors.
\end{abstract}

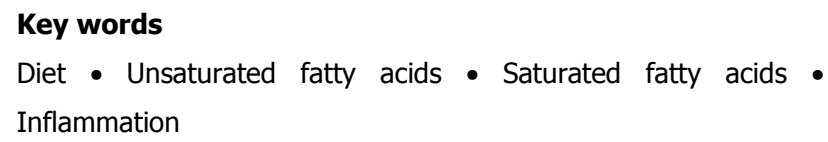

\section{Corresponding author}

I. Králová Lesná, Institute for Clinical and Experimental
Medicine, Vídeňská 1958/9, 14021 Prague 4, Czech Republic. E-mail: ivka@ikem.cz

\section{Introduction}

Atherosclerosis is characterised at the final stage by plaques infiltrated with leucocytes and foam cells. Although an increase of LDL-C concentrations in the intravasal space remains the most important risk factor of the disease, inflammatory mechanisms have gained tremendous research interest over the last two decades (Hanson and Libby 2006). A diet high in saturated fatty acids has been proven to participate in the long lasting process of plaque development and its rupture (Expert Panel 2001). A number of studies have been published over recent years providing evidence that markers of inflammation are strong predictors of atherosclerosis and cardiovascular disease. These markers include proinflammatory cytokines, selectins and acute phase proteins (CRP, procalcitonin etc.) (Ridker et al. 1998a, Malik et al. 2001, Blake and Ridker 2003). Moreover, a CRP increase further deteriorates the negative predictive value of dyslipidemia (Ridker et al. 1998b). The level of subclinical inflammation may be affected by several genetic and environmental factors (Shen and Ordovas 2009) including the effect of individual diet and these factors have been studied as a source of subclinical inflammation during recent years. There is considerable evidence that the visceral fat obesity plays a crucial role 
in the inflammatory process as a chronic inflammatory disease with dysregulation of pro- and antiinflammatory cytokine production (Lyon 2003).

Over the past 30 years, it has become more and more evident that not only the quantity of fat is important for the metabolic consequences of high fat diet but also the quality of fats, i.e. fatty acid composition, is critical for cardiovascular complication progress. The balance of saturated and unsaturated fatty acids and partially the size of the chain are considered as the most important factors influencing bioactivity of different types of fats. The negative effect of a SAFA rich diet was clear from first studies half century ago and was attributed mainly to the increase of total and LDL-C. Additionally, it has been shown that SAFA might directly affect the proinflammation status in adipose tissue (Suganami et al. 2005) and this effect was documented in both monocytes/macrophages and adipocytes. An opposite effect of SAFA and PUFA was demonstrated recently (Han et al. 2010) and key step seems to be NFkB activation resp. inhibition induced by SAFA or PUFA (Lee et al. 2004).

The molecular mechanisms of the direct effect of SAFA to the inflammatory process have been described in detail over recent years. Toll-like receptor 4 (TLR-4), a surface receptor for lipopolysaccharides (LPS), plays a pivotal role in this process by stimulating the production of proinflammatory cytokines (Suganami et al. 2005). Recent results of Schwartz et al. (2010) have definitively presented that LPS are a necessary co-factor of the TLR-4 receptor stimulation. As LPS are in a low concentration presented in the circulation anyway (as a product of metabolism of bacterial flora in the gut), the validity of original work of Suganami et al. (2005) was definitively proven. Thus, TLR-4 activation by SAFA increases the expression of a number of proinflammatory genes (interleukin-6, interleukin-8, etc.) by a NFkB dependent mechanism.

It is therefore highly probable that the high intake of SAFA in the human diet may be proatherogenic also due to stimulation of adipose tissue inflammation by recruitment of monocytes /macrophages in adipose tissue and by induction of proinflammatory changes in trapped macrophages and adipocytes (Han et al. 2010). In spite of information obtained mostly from animal models or in vitro studies, an interventional human clinical study aimed at the effect of a high PUFA diet on subclinical inflammation has shown very inconsistent results (King et al. 2003, Baer et al. 2004, Mozaffarian et al. 2004 etc.).
Stimulated by research data on literature of in vitro SAFA effect on proinflammatory status, we decided to analyze the CRP concentration in frozen samples from our previous project analyzing dietary fatty acids effect on reverse cholesterol transport (Kralova Lesna et al. 2008). This study, performed with young male volunteers, elicited a lower CRP concentration after the PUFA diet compared to the SAFA diet which did not reach statistical significance $(\mathrm{p}=0.056)$. The aim of presented study was to analyse, whether the improvement of experiment control and the amplification of change of the SAFA/PUFA ratio can per se induce significant changes of this marker of subclinical inflammation with a more sensitive model, i.e. middle-aged overweight women.

\section{Methods}

\section{Subjects}

Fifteen women, all employees of the Institute, were included in the study. Only subjects meeting the following criteria were allowed to enrol: dyslipidemic, overweight, aged more than 45 years, and at the same time no evidence of metabolic syndrome or other metabolic diseases (such as diabetes mellitus or thyroid gland dysfunction, etc.). In addition, subjects with evidence of an inflammatory disease or on chronic use of analgesics with antiinflammatory effect were excluded. As soon as informed consent was obtained, the subjects were asked to submit blood samples for introducing analysis of biochemical, immunological and lipid parameters.

\section{Study design and diets}

The study had a randomised crossover design. Two 3-week interventions based on two isocaloric $(11500 \mathrm{~kJ} /$ day $)$ diets were provided to participants and were carried out in succession with a 1 week of washout period - a diet high in saturated fatty acids (SAFA diet) or diet high in polyunsaturated fatty acids (PUFA diet).

The diets composition was completely designed and prepared by registered dieticians and the meals consumed throughout the study were prepared at the Institute facilities. Complete daily meals were provided to participants (breakfast, a mid-morning snack, lunch, an afternoon snack and dinner). The participants were asked not to consume any additional other food during the study, but they were allowed to adjust their food intake to feel comfortable on condition that it will be reduced at 
expense of all parts of served food. Further individualised size portions were balanced weekly to keep stable body weight. Subjects were asked to record any event that could influence the outcome of the study, particularly illnesses, and this aspect was consulted in weekly intervals with physician. Both diets contained a similar amount of protein (13 energy \% in PUFA diet and $12 \%$ in PUFA diet respectively) and carbohydrates (47\% in PUFA and $46 \%$ in SAFA diet). PUFA diet elicited $40 \%$ energy in total fat (ranging from $37 \%$ to $43 \%$ ) and SAFA diet $42 \%$ (ranging from $38 \%$ to $45 \%$ ). Both diets were constructed to reach rather extreme differences in proportion of the SAFA or PUFA diet. Average content of saturated FA in the SAFA diet was $80 \mathrm{~g}(29 \%)$ whereas the content of unsaturated fatty acids was only

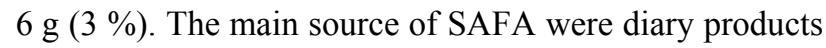
whereas the main source of PUFA were vegetable oils. The PUFA diet had $23 \mathrm{~g}$ of saturated FA $(8.5 \%)$ and $66 \mathrm{~g}$ $(25 \%)$ of polyunsaturated FA. Diet adherence and the subjects compliance was monitored twice a week during repeated sessions with a nutrition specialist when body weight was checked. Subjects were encouraged to maintain their regular physical activities and life style throughout the study and to report departures from their normal pattern of exercise. The consumption of alcohol was not prohibited up to maximal daily dose of $20 \mathrm{~g}$.

After an introductory screening, 15 participants were enrolled in the study. One subject dropped out due subfebrilias lasting 5 days and 14 subjects completed 7 weeks lasting study protocol. One of these participants had a transient viral infection during the PUFA study period and this subject was excluded from the evaluation of this diet period. The other subjects finished the study protocol without any self-monitored change of health.

\section{Anthropometric data}

Height, weight, waist circumference and body composition were determined at the beginning and at the end of each dietary period. Weight was measured twice a week with an accuracy of $0.01 \mathrm{~kg}$. Body composition and basal metabolic rate were determined by an impedance analysis using a Bodystat analyser (1500 MDD; Bodystat, Isle of Man, UK). A trained nurse performed all anthropometric measurements.

\section{Biochemical data}

Blood samples were obtained after a 12-h fasting and collected at the beginning and at the end of both dietary periods. Blood samples for immunological parameters were obtained and analysed twice every time on two consecutive days. Samples were allowed to coagulate at room temperature, separated and frozen to $-80{ }^{\circ} \mathrm{C}$ until analysis at the end of the study. Cholesterol and triglyceride in serum and very low density lipoproteins (VLDL) were determined in the laboratory under the permanent control of the Center for Disease Control, Atlanta, USA with commercial kits (Roche diagnostics, Switzerland, Basel) using an Cobas Mira Plus autoanalyser (Roche, Switzerland, Basel). The high density lipoprotein cholesterol (HDL-C) concentration was measured after phosphotungstate precipitation of apolipoprotein B (Apo B)-containing lipoproteins and low density lipoprotein cholesterol (LDL-C) was measured by direct method using a commercially available kit (Roche diagnostics, Basel, Switzerland) and also calculated with the Friedewald formula (Friedewald et al. 1972).

VLDL were isolated from serum by ultracentrifugation (Havel et al. 1955) and stored at $-80^{\circ} \mathrm{C}$ for analysis of apo $\mathrm{B}$ and fatty acid spectrum at the end of the experiment. Apo B in VLDL was determined using ELISA kit (Abcam plc, Cambridge, UK).

\section{Immunological parameters}

IL-18 was determined using ELISA test (R\&D Systems, Minneapolis, MN). CRP was measured by immunoturbidimetric assay using an autoanalyser (Cobas Mira Plus, Roche, Switzerland, Basel). The serum was obtained exactly as described for biochemical analyses. IL-18 and CRP concentration were determined in two consecutive days at the beginning and at the end of each dietary period. The average of these two measurements was used for calculation to reduce the day to day variability of immunologic data. IL-1 $\beta$, IL- 6 a TNF- $\alpha$ were analysed by Luminex ${ }^{\circledR}$ Assay kits (R\&D Systems, Minneapolis, MN). A high number of these cytokine concentration analyses were below the detectable range in several subjects making a correct analysis of the different dietary effects unpractical.

\section{Analysis of fatty acid spectrum in VLDL particles}

VLDL lipids were extracted according to Folch et al. (1957) and an analysis of triglyceride fatty acids was then carried out by gas chromatography as described by Eder (1995). Briefly, VLDL lipids were separated by thin layer chromatography using hexane-diethyletheracetic acid $(80: 20: 3, \quad \mathrm{v} / \mathrm{v})$ as a solvent system and 
triglycerides were then transmethylated with methanol. Gas chromatography of the fatty acid methyl esters was performed on a GS 5890A instrument (Hewlett Packard, USA) equipped with a flame-ionization detector. A carbowax fused silica capillary column (25 m x $0.25 \mathrm{~mm}$ i.d.) was used. The column temperature was from 150 to $225{ }^{\circ} \mathrm{C}$ (temperature increase $2{ }^{\circ} \mathrm{C} / \mathrm{min}$ ) and hydrogen was used as the carrier gas. Individual peaks of fatty acid methyl ester were identified by comparing retention times with those of authentic standards (Sigma, Czech Republic). The proportional composition/ representation of triglyceride fatty acids (spectrum of 17 main fatty acids) was analyzed.

\section{Ethics aspects}

The local Ethics Committee approved the study protocol according to the Helsinki declaration of 1975 as revised in 2000 and study was conducted in accordance with the approved protocol. All participants were informed about the purpose of the study and the provided written informed consent prior to enrolment in the study.

\section{Statistical analysis}

Results are expressed as mean \pm standard deviation (SD). The differences between SAFA and PUFA dietary periods were evaluated using a paired t-test and for trend of CRP concentration changes Wilcox test was used.

\section{Results}

\section{Subjects and diets}

Waist circumference did not differ significantly when the basal and final data of both diet periods were compared $(99.27 \pm 7.99 \mathrm{~cm}$ and $98.20 \pm 7.77 \mathrm{~cm}$ for PUFA diet and $98.67 \pm 7.15 \mathrm{~cm}$ and $7.41 \mathrm{~cm}$ for SAFA diet respectively). Starting BMI $\left(31.63 \pm 4.14 \mathrm{~kg} . \mathrm{m}^{-2}\right)$ did not change during the experiment significantly. The average value of BMI with waist circumference $99.27 \pm 7.88 \mathrm{~cm}$ documented that obese individuals with central type of obesity were enrolled for this experiment. Adiposity tissue content did not change significantly neither in the SAFA diet (basal 35.5 $\pm 7.9 \%$ and final $35.4 \%$ ) nor in the PUFA diet (basal 35.7 $\pm 7.12 \%$ and final $35.2 \pm 7.5 \%$ ). All individuals were able to keep their body weight within the range $1.5 \mathrm{~kg}$ through all experiment.

\section{Changes of lipoprotein parameters}

Adherence to both dietary regimens was documented by changes of fatty acids spectrum in VLDL fraction. The size and composition of VLDL particles were not changed when basal and final data of VLDL concentration and VLDL triglycerides were compared (Table 1). The cholesterol/triglyceride ration ranged around 0.4 during whole experiments. The only significant change was a small decrease of VLDL apoB concentration after PUFA diet for about $20 \%$. Although changes of individual fatty acid proportion in VLDL triglycerides during each dietary period did not reach statistical significance, there was highly significant difference in the sum of SAFA and PUFA content when the final data of both dietary regimens were compared (Table 1). As expected, no significant changes in the proportion of MUFA during the dietary changes were found. On the contrary, the proportion of SAFA was for $20 \%$ higher at the end of SAFA diet compared to PUFA diet $(\mathrm{p}<0.02)$, the most pronounced difference was found in the proportion of the $\omega 6$ PUFA sum which was exceedingly significantly increased after the PUFA diet $23.32 \pm 3.28$ compared to $18.62 \pm 3.46$ after the SAFA diet $(\mathrm{p}<0.001)$. Although changes in 63 PUFA were not so marked, the difference reached statistical significant.

Two subgroups of both branches of cross-over setting did not differ in the concentration of lipoproteins at the very beginning of the study of the experiment. Expected changes of total cholesterol and LDL cholesterol after the PUFA or SAFA diets were confirmed. The PUFA diet resulted in significant $14 \%$ $(\mathrm{p}<0.01)$ decrease of total cholesterol, $9.5 \%$ decrease of LDL-C $(\mathrm{p}<0.01)$ and $11 \%(\mathrm{p}<0.05)$ decrease in HDL-C whereas TG did not change when compared to the baseline values. An opposite trend could be observed for the SAFA diet, where the total cholesterol increased for $11.5 \%(\mathrm{p}<0.001)$, LDL-C increased for $12.5 \%(\mathrm{p}<0.01)$, while HDL and TG did not changed substantially. When the final values from both diet regimes are compared, highly significant differences of total cholesterol $(\mathrm{p}<0.001)$, LDL-C $(\mathrm{p}<0.001)$ and HDL-C $(\mathrm{p}<0.01)$ were found (Table 2). The pattern of LDL-C concentration changes induced by both dietary regimens was the same using the direct measurement or Friedewald formula calculation (calculated data display slightly lower value).

\section{Effect of diets on immunological parameters}

C-reactive protein significantly decreased $(\mathrm{p}<0.02)$ after the PUFA diet period $(6.61 \pm 2.49$ vs. $2.56 \pm 1.64 \mathrm{mmol} / \mathrm{l}, \mathrm{p}<0.05)$, whereas its increase after the SAFA diet did not reach statistical significance $(2.95 \pm 1.98$ vs. $3.30 \pm 2.54 \mathrm{mmol} / \mathrm{l}$, n.s.). As there were 
Table 1. VLDL composition.

\begin{tabular}{|c|c|c|c|c|c|c|c|c|}
\hline $\mathbf{a}$ & VLDL-C & VLDL-TG & VLDL C/TG & VLDL-ApoB & SAFA & MUFA & PUFA $\omega 6$ & PUFA $\omega 3$ \\
\hline basal PUFA & 0.37 & 0.89 & 0.43 & 31.17 & 24.30 & 52.95 & 21.04 & 1.70 \\
\hline$S D$ & 0.21 & 0.62 & 0.05 & 11.39 & 4.50 & 3.54 & 3.53 & 0.98 \\
\hline after PUFA & 0.44 & 1.05 & 0.43 & 23.16 & 21.85 & 52.91 & 23.32 & 1.87 \\
\hline$S D$ & 0.28 & 0.70 & 0.05 & 5.40 & 7.37 & 5.26 & 3.28 & 0.92 \\
\hline basal SAFA & 0.41 & 1.10 & 0.38 & 32.23 & 22.11 & 54.78 & 21.34 & 1.76 \\
\hline$S D$ & 0.22 & 0.63 & 0.08 & 16.43 & 6.48 & 4.61 & 4.24 & 0.95 \\
\hline $\begin{array}{l}\text { after } S A F A \\
S D\end{array}$ & $\begin{array}{l}0.48 \text { n.s. } \\
0.30\end{array}$ & $\begin{array}{l}1.18 \text { n.s. } \\
0.83\end{array}$ & $\begin{array}{l}0.42 \text { n.s. } \\
0.09\end{array}$ & $\begin{array}{l}33.29 \text { n.s. } \\
15.07\end{array}$ & $\begin{array}{l}26.01 \text { n.s. } \\
4.98\end{array}$ & $\begin{array}{l}53.89 \text { n.s. } \\
3.70\end{array}$ & $\begin{array}{l}18.62 \text { n.s. } \\
3.46\end{array}$ & $\begin{array}{l}1.26 \text { n.s. } \\
0.33\end{array}$ \\
\hline \multicolumn{9}{|l|}{ b } \\
\hline$P U F A x S A F A$ & 0.503 & 0.231 & 0.698 & 0.402 & $\mathrm{p}<0.02$ & 0.401 & $\mathrm{p}<0.001$ & $\mathrm{p}<0.02$ \\
\hline
\end{tabular}

a: comparison of basal and final data of PUFA and SAFA diet resp. (concentration of VLDL C and VLDL TG in $\mathrm{mmol} / \mathrm{l}, \mathrm{VLDL}$ apoB in $\mathrm{mg} / \mathrm{l}$, fatty acid composition is expressed in mol\%), b: comparison of effects of both diets at the end of experimental period.

Table 2. Changes of lipoprotein and glucose concentrations.

\begin{tabular}{lllllll}
\hline & TC & TG & HDL-c & LDL-c & LDL-c F & Glucose \\
\hline basal PUFA & 5.99 & 1.73 & 1.44 & 3.30 & 3.76 & 5.32 \\
SD & 0.94 & 0.87 & 0.98 & 0.86 & 0.89 & 0.54 \\
after PUFA & $5.15 \mathrm{~b}$ & $1.95 \mathrm{n} . \mathrm{s}$. & $1.28 \mathrm{c}$ & $2.99 \mathrm{~b}$ & $3.33 \mathrm{a}$ & 5.23 n.s. \\
SD & 0.94 & 0.96 & 0.39 & 0.78 & 0.87 & 0.91 \\
basal SAFA & 5.87 & 1.91 & 1.39 & 3.29 & 3.64 & 5.54 \\
SD & 0.64 & 0.97 & 0.38 & 0.57 & 0.73 & 0.58 \\
after SAFA & $6.54 \mathrm{a}$ & $1.96 \mathrm{n} . \mathrm{s}$. & $1.42 \mathrm{n} . \mathrm{s}$. & $3.70 \mathrm{~b}$ & $4.22 \mathrm{a}$ & 5.32 n.s. \\
SD & 0.90 & 0.97 & 0.43 & 0.78 & 0.91 & 0.54 \\
PUFAxSAFA & $\mathrm{p}<0.001$ & n.s. & $\mathrm{p}<0.01$ & $\mathrm{p}<0.001$ & $\mathrm{p}<0.001$ & n.s. \\
\hline
\end{tabular}

Concentration of all lipoprotein fraction and glucose is expressed in mmol/l. Statistical significance comparing basal concentration and after PUFA or SAFA diet respectively $-p<0.001-a, p<0.01-b, p<0.05-c$. Significance of comparison PUFA and SAFA diet at the end of the period is expressed at the bottom of the table.

substantial differences in basal values of CRP concentration interindividually (minimal CRP concentration $0.32 \mathrm{mmol} / \mathrm{l}$, maximal concentration $7.48 \mathrm{mmol} / \mathrm{l})$ it is optimal express changes of this proinflammatory parameter as percentage changes of individual values due to data normalization. When the trend of individual changes of CRP concentration compared to both the PUFA and SAFA diets was calculated, a significant difference $(\mathrm{p}<0.01)$ was found (Fig. 1). We could demonstrate a decrease of interleukin 18 after the PUFA diet $(\mathrm{p}<0.05)$, but a change after the SAFA diet was not observed. We failed to statistically evaluate other immunological parameters (TNF- $\alpha$, IL-1 $\beta$ and IL-6) as levels of these cytokines balanced on the detectable level of the analytical kits used.

\section{Discussion}

In this study, we have proven that a switch to a polyunsaturated fatty acids diet can decrease a well known proinflammatory marker, i.e. C-reactive protein. As CRP has been repeatedly confirmed (Ridker et al. 1998b) to be a cardiovascular risk factor, we suppose that there is an additional important direct effect of these types of fatty acids.

Over the last decade, the orientation of atherosclerosis pathology research diverted from traditional risk factors to metabolic/inflammation processes. Dietary intake of saturated fatty acids probably effects the atherosclerosis process in both indirect and direct ways. Its indirect effect in increasing LDL 


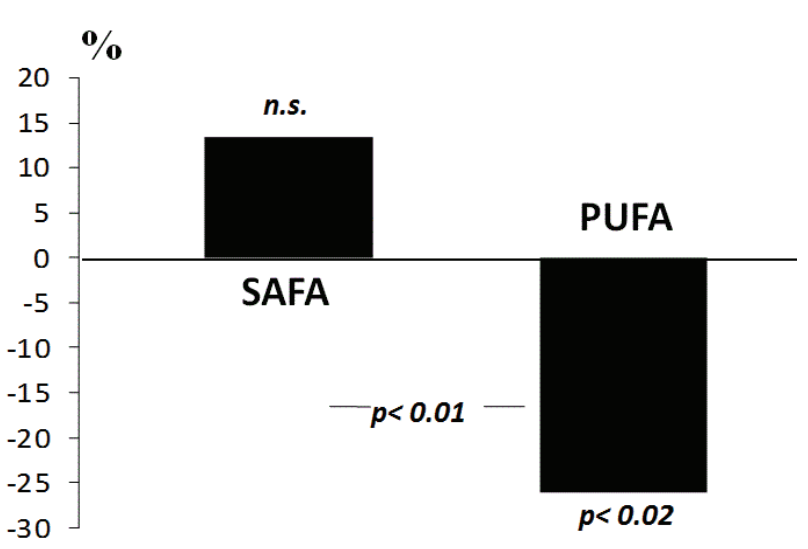

Fig. 1. CRP concentrations are expressed in \% changes when basal with data at the end of each experimental period. The increase of CRP after SAFA diet did not reached statistical significance whereas the decrease after PUFA diet was significant. The trend of SAFA and PUFA diet comparison calculated by Wilcox analysis is highly significant.

concentration has been repeatedly proven and also molecular biological mechanism of this effect is well known. Although the direct effect of SAFA in vivo in men has not been proven by epidemiology and clinical data definitely, the molecular biology mechanism of the proinflammatory effect of SAFA has been described at the tissue cultures level (Suganami et al. 2009). Perhaps the reason for the incomplete proof of the direct effect of the SAFA diet on inflammation in humans to date is due to methodological problems. In large epidemiological studies, a correct analysis of total fat and proportion of SAFA in diet is complicated by inaccuracy of all methods which are used. Food frequency and a one day dietary record are not very accurate tools but this approach is only possible when applied in large studies. The alternative approach - controlled clinical studies are usually limited in size and accuracy in order to strictly fulfil protocol of the study. In addition, another limitation of these types of studies is the genetic both to basal and experimentally affected CRP concentration (Poledne et al. 2009, Shen and Ordovas 2009).

It should be stated that the response rate and behaviour of our volunteers was very good. It was influenced by their selection from employees of our Institute, clerks and nurses, which have been employees for many years with long lasting contact with cardiovascular disease research. In cooperation with our Institute kitchen, the correct diet composition and delivery to volunteers was carried out daily. A full day meal with exactly defined composition was carefully prepared according to instructions of nutritionist. Related to body weight changes analysed (a minimum twice weekly), detail acute changes in energy intake were realised and it maintained body weight changes within limit $\pm 1.5 \mathrm{~kg}$ throughout the study.

As it is documented (Table 1), no substantial changes of VLDL particles structure appeared, a slight decrease in the number of VLDL particles after the PUFA diet was found (ApoB concentration decreased. In contrast, the composition fatty acids in VLDL particles PUFA and SAFA proportion changed. The expected increase of the proportion of PUFA (both $\omega 3$ and $\omega 6$ ) appeared after PUFA diet (for $15 \%$ ) and opposite changes appeared after the SAFA diet (SAFA content increased for $20 \%$ whereas PUFA content dropped at similar proportion). Due to high individual variability, none of these changes reached statistical significance. When these changes were compared as differences analysed at the end of period with PUFA and SAFA respectively, significant changes were documented both in total SAFA and $\omega 3$ and $\omega 6$ PUFA (Table 1).

Expected changes of atherogenic lipoprotein concentration were detected. The SAFA diet increased total and LDL cholesterol only with borderline significance, whereas the PUFA diet decreased their concentration highly significantly. It might be explained by the closer similarity of usual diet of our volunteers to the high SAFA diet than to the PUFA diet. A sizable part of dietary fat intake of the Czech population still comes from meat, butter and other dairy products (Annual Reports of the Institute of Agricultural Economics and Information of the Czech Republic). It is important to stress that VLDL particles in the circulation do not supply fatty acids to all peripheral tissue (either in fasting or postprandially) due to competition with chylomicrons as carriers of dietary lipids for lipoprotein lipase despite the fact that the majority of triglycerides in postprandial plasma is composed of VLDL (Kovář and Havel 2002). Changes of VLDL fatty acids composition induced during only 3 weeks of the SAFA diet period might be more pronounced after a life-long unhealthy diet.

Since our 3-weeks model experiment induced changes of systemic inflammation, it is highly probable that a long lasting diet with a high proportion of unsaturated fats may have a positive effect on the incidence of cardiovascular risk. In contrast to the well known increased risk of dietary induced hypercholesterolemia to cardiovascular disease mortality discovered half century ago, the effect of inflammation was proven merely a decade ago (Ridker et al. 1998a). Recently, the proinflammatory effect of saturated fatty 
acids was well described in macrophages as well as in adipocytes. SAFA (namely more palmitate, laurate and myristate than stearate) are able to stimulate many inflammatory genes through the TLR-4 receptor in coordination with lipopolysaccharides (Han et al. 2010). Although this mechanism of SAFA initiating proinflammatory status of different cells in culture is well known in detail (Lee et al. 2004), and there are plausible biological mechanisms for direct proinflammatory effect of SAFA, the in vivo effect of SAFA on different inflammatory markers is still very controversial issue.

Out of numerous markers of inflammation used in epidemiology and clinical studies, C-reactive protein has been used the most frequently as a marked parameter of inflammatory changes. Out of markers measured in this study, only interleukin 18 and CRP concentration changes were detected. Although changes of IL 18 concentration did not reach statistical significant when the basal and SAFA diet were compared, and only a borderline significance of the PUFA diet change was detected, the pattern of changes was similar to changes of CRP concentration. Several studies analyzing the possible effect of changes in dietary fatty acid composition on proinflammatory status were published with rather controversial results. It is very probably influenced by different methodologies used. Two different methodological approaches have been used. An analysis of large population samples using a relatively simple one day dietary record and well controlled clinical studies with a rather limited numbers of volunteers included. It is evident that an analysis of a long lasting diet with one day dietary record is not very accurate (Suchanek et al. 2011) and the proinflammatory effect of SAFA was not found in Mozaffarian study (Mozaffarian et al. 2004) based on subsamples of the Nurse Health Study. Only systemic inflammation was accented by an increased intake of trans-fatty acids in the above mentioned study. This effect was confirmed by a well controlled randomised cross over study by Baer (Baer et al. 2004), who further found that diet enriched with 12 : and 16: fatty acids influence proinflammatory status measured not only by CRP but also by IL6 concentration. More well-controlled clinical studies in volunteers are needed to follow metabolic changes of different diet with higher SAFA or PUFA content to influence biomarkers in vivo.

In our experiment, the proinflammatory effect of SAFA diet was smaller compared to antiinflammatory effect of PUFA diet. This may be due to dietary habits of Czech population with still too high intake of animal fat (data from our epidemiological study, not presented). It is with agreement with data of Zempelas et al. (2005) documenting that CRP concentration was lower in individuals consuming more fish.

The result of this study proved that proportion of PUFA and SAFA the dietary intake might affect the systemic inflammation and to influence CVD risk in addition to a change of concentration of atherogenic lipoproteins.

\section{Conflict of Interest}

There is no conflict of interest.

\section{Acknowledgements}

Supported by the project (Ministry of Health, Czech Republic) for development of research organization 00023001 (IKEM, Prague, Czech Republic) Institutional support.

\section{References}

BAER DJ, JUDD JT, CLEVIDENCE BA, TRACY RP: Dietary fatty acids affect plasma markers of inflammation in healthy men fed controlled diets: a randomized crossover study. Am J Clin Nutr 79: 969-973, 2004.

BLAKE GJ, RIDKER PM: C-reactive protein and other inflammatory risk markers inacute coronary syndromes. $J$ Am Coll Cardiol 41: 37S-42S, 2003.

EDER K: Gas chromatographic analysis of fatty acid methyl esters: $J$ Chromatogr B Biomed Appl 15: 113-131, 1995.

EXPERT PANEL: Executive summary of the third report of the National Cholesterol Education Program (NCEP) Expert Panel on detection, evaluation, and treatment of high blood cholesterol in adults (Adult Treatment Panel III). JAMA 285: 2486-2497, 2001.

FUNG TT, RIMM EB, SPIEGELMAN D, RIFAI N, TOFLER GH, WILLETT WC, HU BF: Association between dietary patterns and plasma biomarkers of obesity and cardiovascular disease risk. Am J Clin Nutr 73: 61-67, 2001.

FOLCH J, LESS M, SLOANE STANLEY GH: A simple method for the isolation and purification of total lipids from animal tissues. $J$ Biol Chem 226: 497-509, 1957. 
FRIEDEWALD WT, LEVY RI, FREDRICKSON DS: Estimation of the concentration of low-density lipoprotein cholesterol in plasma, without use of preparative ultracentrifuge. Clin Chem 18: 499-502, 1972.

HAN CY, KARGI AY, OMER M, CHAN CK, WABITSCH M, O'BRIEN WIGHT TN, CHAIT A: Differential effect of saturated and unsaturated free fatty acids on the generation of monocyte adhesion and chemotactic factors by adipocytes: dissociation of adipocyte hypertrophy from inflammation. Diabetes 59: 386-396, 2010.

HANSON GK, LIBBY P: The immune response in atherosclerosis: a double-aged sword. Nat Rev Immunol 6: 508-519, 2006.

HAVEL RJ, EDER HA, BRAGDON JH: The distribution and chemical composition of ultracentrifugally separated lipoproteins in human serum. J Clin Invest 34: 1345-1353, 1955.

KING DE, EGAN BM, GEESEY ME: Relation of dietary fat and fiber to elevation of C-reactive protein. Am J Cardiol 92: 1335-1339, 2003.

KOVÁŘ J, HAVEL RJ: Sources and properties of triglyceride-rich lipoproteins containing apoB-48 and apoB-100 in postprandial blood plasma of patients with primary combined hyperlipidemia. J Lipid Res 43: 1026-1034, 2002.

KRALOVA LESNA I, SUCHANEK P, KOVAR J, STAVEK P, POLEDNE R: Replacement of dietary saturated fatty acids by polyunsaturated fatty acids in diet and reverse cholesterol transport. J Lipid Res 49: 2414-2417, 2008.

LEE JY, ZHAO L, YOUN HS, WEATHERILL AR, TAPPING R, FENG L, LEE WH, FITZGERALD KA, HWANG DH: Saturated fatty acid activates but polyunsaturated fatty acid inhibits Toll-like receptor 2 dimerizied with Toll-like receptor 6 or 1. J Bio Chem 279: 16971-16979, 2004.

LYON CJ, LAW RE, HSUEH WA: Minireview: adiposity, inflammation, and atherogenesis. Review. Endocrinology 144: 2195-2200, 2003.

MALIK I, DANESH J, WHINCUP P, BHATIA V, PAPACOSTA O, WALKER M, LENNON L,THOMSON A, HASKARD D: Soluble adhesion molecules and prediction of coronary heart disease: a prospective study and meta-analysis. Lancet 358: 971-976, 2001.

MOZAFFARIAN D, PISCHON T, HANKINSON SE, RIFAI N, JOSHIPURA K, WILLETT WC, RIMM EB: Dietary intake of trans fatty acids and systemic inflammation in women. Am J Clin Nutr 79: 606-612, 2004.

POLEDNE R, LORENZOVÁ A, STÁVEK P, VALENTA Z, HUBÁČEK J, SUCHÁNEK P, PIŤHA J: Proinflammatory status, genetics and atherosclerosis. Review. Physiol Res 58: S111-S118, 2009.

RIDKER PM, GLYNN RJ, HENNEKENS CH: C-reactive protein adds to the predictive value of total and HDL cholesterol in determining risk of first myocardial infarction. Circulation 97: 2007-2011, 1998a.

RIDKER PM, CUSHMAN M, STAMPFER MJ, TRACY RP, HENNEKENS CH: Plasma concentration of C-reactive protein and risk of developing peripheral vascular disease. Circulation 97: 425-428, 1998b.

SCHWARTZ EA, ZHANG WY, KARNIK SK, BORWEGE S, ANAND VR, LAINE PS, SU Y, REAVEN PD: Nutrient modification of the innate immune response: a novel mechanism by which saturated fatty acids greatly amplify monocyte inflammation. Arterioscler Thromb Vasc Biol 30: 802-808, 2010.

SHEN J, ORDOVAS JM: Impact of genetic and environmental factors on hsCRP concentrations and response to therapeutic agens. Clin Chem 52: 256-264, 2009.

SUCHANEK P, POLEDNE R, HUBACEK JA: Dietary intake reports fidelity - fact orfiction? Neuro Endocrinol Lett 32 (Suppl 2): 29-31, 2011.

SUGANAMI T, NISHIDA J, OGAWA Y: A paracrine loop between adipocytes and macrophages aggravates inflammatory changes: role of free fatty acids and tumor necrosis factor alpha. Arterioscler Thromb Vasc Biol 25: 2062-2068, 2005.

SUGANAMI T, YUAN X, SHIMODA Y, UCHIO-YAMADA K, NAKAGAWA N, SHIRAKAWA I, USAMI T, TSUKAHARA T, NAKAYAMA K, MIYAMOTO Y, YASUDA K, MATSUDA J, KAMEI Y, KITAJIMA S, OGAWA Y: Activating transcription factor 3 constitutes a negative feedback mechanism that attenuates saturated Fatty acid/toll-like receptor 4 signaling and macrophage activation in obese adipose tissue. Circ Res 105: 25-32, 2009.

ZEMPELAS A, PANAGIOTAKOS DB, PITSAVOS D, DAS UN, CHRYSOHOOU C, SKOUMAS Y, STEFANADIS E: Fish consumption among healthy adults is associated with decreased levels of inflammatory markers related to cardiovascular disease: the ATTICA study. J Am Coll Cardiol 46: 120-124, 2005. 